\title{
Bromination of Enamines from Methyl Isopropyl Ketone. A Possible Route to Bromomethyl Isopropyl Ketone
}

\author{
ROLF CARLSON and CHRISTOFFER RAPPE
}

\author{
Department of Organic Chemistry, University of Umeå, S-901 87 Umeå, Sweden
}

The relative amounts of the various bromo
ketones obtained by reacting the morpholine
enamines of methyl isopropyl ketone with
bromine under different conditions were deter-
mined. Mechanistic details of the reaction are
briefly discussed. A convenient method of pre-
paring bromomethyl isopropyl ketone via the
morpholine enamine is described and the opti-
mum conditions for this reaction are presented.

Enamines react with elementary halogens to form $\alpha$-haloimmonium salts, which on hydrolysis yield the corresponding $\alpha$-halocarbonyl compounds. ${ }^{1-7}$ It has also been reported that certain imines from methyl ketones react with $N$ halosuccinimides to yield halomethyl ketones involving enamine intermediates..$^{8,9}$

When an unsymmetric ketone with $\alpha$ - and $\alpha^{\prime}$-hydrogens, e.g. methyl isopropyl ketone, is converted to the corresponding tertiary enamine using a secondary amine, e.g. morpholine, two isomeric tertiary enamine products are possible, I and II (Scheme 1). The isomers I and II are interconvertible and the equilibrium ratio of

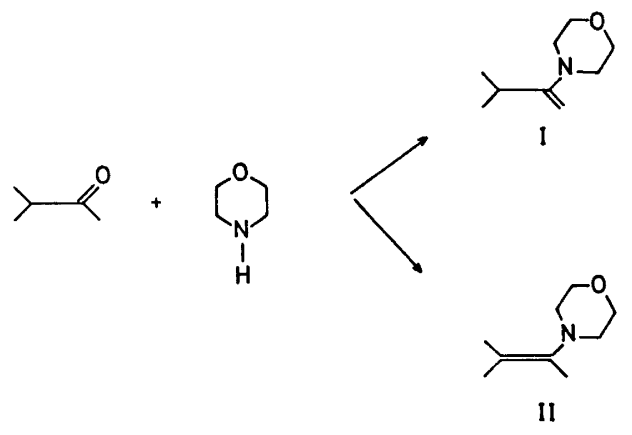

Scheme 1.
I:II is 29:71 at room temperature. However the equilibration is a rather slow process in absence of heat and/or acid, and the isolation of $I$ and II in pure state has been reported..$^{10}$

$\alpha$-Halogenation of ketones occurs in the enolized ketone. When unsymmetric ketones are halogenated, mixtures of isomeric haloketones are usually obtained, due to formation of isomeric enols. In some cases a 1:1 ratio of isomeric $\alpha$-haloketones is reported. ${ }^{11}$ It is difficult to prepare halomethyl ketones by direct halogenation of ketones. However, Gaudry and Marquet have reported that bromination of a number of methyl ketones in carbon tetrachloride containing a few percent of methanol affords high yields of the bromomethyl ketone. ${ }^{12}$

No reports of halogenation of tautomerizable tertiary enamines have been found in the literature. The purpose of the present investigation is to ascertain whether or not transformation of a ketone to the corresponding tertiary enamine offers a means of overcoming difficulties encountered by direct halogenation of the ketone. We wish to report some results obtained by bromination of the morpholine enamines from methyl isopropyl ketone. Results from the bromination of the corresponding dimethylamino and pyrrolidino enamines will be reported later, due to the fact that these experiments led to a negative product balance, and the reason for this is not yet fully understood. Control experiments showed that the recovery of ketone was $92 \%$ and of bromine $96 \%$ when the morpholine enamine $I$ was treated with one equivalent of bromine and subsequently hydrolyzed. The recovery of ketone in experiments with dimethylamino and 
pyrrolidino enamines was considerably lower.

The enamines I, II as well as I+II in equilibrium mixture were allowed to react with 0.5 , 1.0 , and 2.0 equivalents of bromine in methylene chloride at $-78^{\circ} \mathrm{C}$ and room temperature. The reaction between the enamines and bromine is instantaneous and the reaction mixture was quenched with water after one minute followed by removal and analysis (GLC) of the organic layer. The ketones III-VII (Scheme 2) were identified and the results of these experiments are summarized in Table 1.

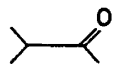

III<smiles>CC(C)C(=O)C(Br)Br</smiles>

VI

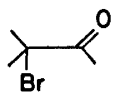

IY

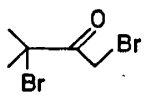

VII
Scheme 2.

These results may be interpreted in the following way. At $-78{ }^{\circ} \mathrm{C}$ the enamines are rapidly converted to the corresponding $\alpha$-bromo- immonium salts, which are relatively stable towards further bromination. Consequently the yields of dibromoketones are low in these experiments. At room temperature the $\alpha$-bromoimmonium salts which constitute the primary products undergo further reaction prior to hydrolysis. The yield of the dibromo ketone VII is rather high when one equivalent of bromine is used and this has led us to propose the intermediates VIII and IX (Scheme 3).

It should be noted that the yield of $\mathrm{V}$ far exceeds the amount of the corresponding enamine I present in the equilibrium mixture and as much as $c a .14 \%$ of $\mathrm{V}$ is found when a pure sample of the other isomer II is brominated. This unexpected result is explained by the reaction sequence in Scheme 3. The dibromoimmonium salt IX is debrominated to the bromo enamine $\mathrm{X}$ which is then protonated or brominated on the nitrogen atom. These cationic species are not prone to react with the electrophile bromine.

This explanation is further supported by time interval measurements carried out on the reaction between the enamines and bromine at room temperature (see Table 2). One equivalent of bromine was used throughout. The table

Table 1. Product distribution obtained by bromination of the enamines under conditions given in the text.

\begin{tabular}{|c|c|c|c|c|c|c|c|c|}
\hline Enamine & Equiv. $\mathrm{Br}_{2}$ & Temp. ${ }^{\circ} \mathrm{C}$ & \multicolumn{6}{|c|}{ Products after hydrolysis (\%) } \\
\hline $\mathrm{I}^{a}$ & 0.55 & -78 & 45 & - & 55 & - & - & - \\
\hline $\mathrm{I}^{a}$ & 0.55 & Rt & 47 & - & 53 & - & - & - \\
\hline $\mathrm{I}^{a}$ & 1.09 & -78 & 2 & 8 & 90 & $<0.5$ & - & trace \\
\hline $\mathrm{I}^{a}$ & 1.09 & Rt & 6 & $<0.5$ & 84 & 0.5 & 9 & trace \\
\hline$I^{b}$ & 2.0 & -78 & 5 & 7 & 85 & 1 & 2 & $<1$ \\
\hline $\mathbf{I}^{b}$ & 2.0 & $\mathrm{Rt}$ & 2 & 6 & 88 & 1 & 2 & $<1$ \\
\hline $\mathrm{II}^{c}$ & 0.5 & -78 & 42 & 56 & 1 & - & 1 & - \\
\hline $\mathrm{II}^{c}$ & 0.5 & Rt & 80 & 17 & trace & - & 1 & 1 \\
\hline $\mathrm{II}^{c}$ & 1.0 & -78 & 1 & 95 & 1 & - & 3 & - \\
\hline $\mathrm{II}^{c}$ & 1.0 & Rt & 29 & 11 & 14 & - & 46 & $<0.5$ \\
\hline$\overline{\mathrm{II}}^{c}$ & 2.0 & -78 & trace & 92 & 1 & - & 7 & trace \\
\hline $\mathrm{II}^{c}$ & 2.0 & Rt & trace & 81 & 1 & - & 18 & 1 \\
\hline$\overline{\mathrm{I}}+\mathrm{II}^{d}$ & 0.5 & -78 & 49 & 26 & 24 & - & - & I \\
\hline $\mathrm{I}+\mathrm{II}^{d}$ & 0.5 & Rt & 70 & 5 & 23 & - & - & 2 \\
\hline $\mathrm{I}+\mathrm{II}^{d}$ & 1.0 & -78 & 2 & 64 & 27 & trace & 8 & trace \\
\hline $\mathrm{I}+\mathrm{II}^{d}$ & 1.0 & Rt & 19 & 8 & 47 & - & 26 & $<0.5$ \\
\hline $\mathrm{I}+\mathrm{II}^{d}$ & 2.0 & -78 & 2 & 67 & 27 & - & 3 & - \\
\hline $\mathrm{I}+\mathrm{II}^{d}$ & 2.0 & $\mathbf{R t}$ & 2 & 53 & 27 & trace & 19 & - \\
\hline
\end{tabular}

$a_{1: \mathrm{II}=89.5: 10.5 .}{ }^{b} \mathrm{I}: \mathrm{II}=91.0: 9.0$. Determined from NMR spectrum ${ }^{c}$ No trace of $\mathrm{I}$ was detected in NMR spectrum. ${ }^{d}$ Equilibrium mixture $\mathrm{I}: \mathrm{II}=29.0: 71.0{ }^{e}$ Unidentified peaks in the gas chromatogrammes. 
<smiles>[10BH2]</smiles><smiles></smiles>

YIII<smiles>C=C(N1CCOCC1)C(C)(C)Br</smiles>

$\stackrel{\mathrm{Br}_{2}}{\longrightarrow}$

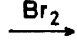

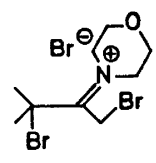

IX
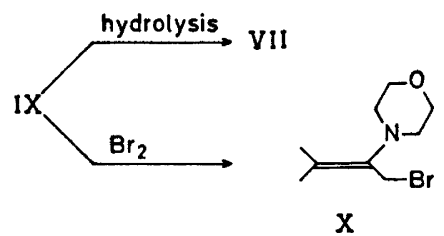

Scheme 3.

Table 2. Time interval measurements of product distribution obtained by bromination of the enamines with one equivalent of bromine at room temperature.

\begin{tabular}{|c|c|c|c|c|c|c|c|}
\hline Enamine & $\begin{array}{l}\text { Time } \\
\text { min }\end{array}$ & \multicolumn{4}{|c|}{ Products after hydrolysis $(\%)$} & VII & Others \\
\hline$I^{a}$ & $\begin{array}{r}1 \\
30 \\
1440\end{array}$ & $\begin{array}{l}19 \\
13 \\
17\end{array}$ & $\begin{array}{r}1 \\
3 \\
12\end{array}$ & $\begin{array}{l}54 \\
61 \\
57\end{array}$ & $\begin{array}{l}\text { trace } \\
\text { trace } \\
-\end{array}$ & $\begin{array}{l}26 \\
22 \\
13\end{array}$ & $\frac{-}{2}$ \\
\hline $\mathrm{II}^{b}$ & $\begin{array}{r}1 \\
30 \\
1440\end{array}$ & $\begin{array}{l}31 \\
30 \\
29\end{array}$ & $\begin{array}{r}6 \\
2 \\
11\end{array}$ & $\begin{array}{r}8 \\
30 \\
32\end{array}$ & $\begin{array}{l}\text { trace } \\
-\end{array}$ & $\begin{array}{l}56 \\
37 \\
19\end{array}$ & $\begin{array}{l}1 \\
1 \\
9^{d}\end{array}$ \\
\hline $\mathrm{I}+\mathrm{II}^{c}$ & $\begin{array}{r}1 \\
30 \\
1440\end{array}$ & $\begin{array}{l}21 \\
20 \\
22\end{array}$ & $\begin{array}{r}5 \\
3 \\
13\end{array}$ & $\begin{array}{l}\mathbf{3 7} \\
\mathbf{5 0} \\
\mathbf{4 7}\end{array}$ & $\begin{array}{l}\operatorname{trace} \\
\text { trace } \\
-\end{array}$ & $\begin{array}{l}36 \\
27 \\
10\end{array}$ & $\begin{array}{r}<1 \\
1 \\
5\end{array}$ \\
\hline
\end{tabular}

${ }^{a} \mathrm{I}: \mathrm{II}=85: 15 .{ }^{b} \mathrm{I} 98 \%{ }^{c}$ Equilibrium mixture $\mathrm{I}: \mathrm{II}=29: 71 .{ }^{d}$ Distributed over seven minor peaks in the gas chromatogramme. ${ }^{e}$ Unidentified peaks in the gas chromatogrammes.

clearly shows that for the dibrominated product the bromination is reversible under these conditions.

When two equivalents of bromine are used the yields of dibrominated ketones are low which might be explained by rapid $N$-bromination of the bromo enamines formed in the reaction mixture when the primarily formed monobromo immonium salts are deprotonated. This indicates that bromination of the nitrogen atom proceeds more rapidly than bromination of the $\beta$-carbon in bromo enamines. Excess bromine enables $N$-bromination go almost to completion and consequently the concentration of free bromo enamine susceptible to attack of bromine on the $\beta$-carbon is low.
Yields of the 1,1-dibromoketone VI are very low in all experiments. However, spiking experiments showed that this is not due to a rearrangement of VI to VII during hydrolysis and work up. The low yields of dibromoketones from the bromination of I can probably be explained on the basis of a more rapid proton transfer from the methyl group in the immonium salt VIII, than from the bromomethyl group or from the isopropyl group in XI (Scheme 4). Therefore

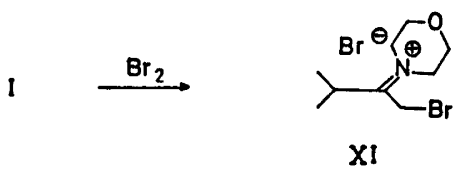

Scheme 4.

Acta Chem. Scand. B 28 (1974) No. 9 
we assign the amounts of VII as coming from the minor amounts of II present in the starting enamine. The proton is transferred to an unbrominated enamine which is consequently withdrawn from further bromination by this protonation. The amounts of III are somewhat too small to account for this proton transfer, but some parent ketone is apparently lost during hydrolysis and work up. The slower proton transfer from XI than from VIII is probably due to steric hindrance in the base attack.

Table 1 shows that it is possible to prepare bromomethyl isopropyl ketone $\mathrm{V}$ in a high yield by the reaction of the least substituted enamine I with bromine. The experiments accounted for in Table 1 were performed on a 10 mmolar scale and we have also tried to discover the optimum conditions for synthesis on a larger scale. In this context some experimental findings are of importance. In the experiments listed in Tables 1 and 2 the enamine was added to a stirred solution of bromine. The reverse addition procedure is unsuitable since it leads to considerable amounts, approximately $40 \%$, of unbrominated ketone, in addition to a rather high yield of the dibromomethyl ketone VI. This can be explained by a proton transfer from the monobromo immonium salt to an unbrominated enamine, followed by a second bromination of the bromo enamine thus formed. This can be avoided by the addition of enamine to bromine. A direct scale-up also yielded rather large amounts of dibromo ketones, and it is possible that this may be caused by heat evolved during the reaction.

The enamine $I$ is the kinetically controlled product when it is prepared according to the method described by White and Weingarten. ${ }^{13}$ In this reaction hydrocarbons are preferred as solvents, and using pentane we attempted a direct bromination of the crude enamine. However, it was found to give rise to practical difficulties. The bromo immonium salts formed precipitated as a sticky mass which made stirring impossible. However, methylene chloride is known to be a good solvent for ion pairs ${ }^{14}$ and we found it to be a more favourable solvent. Use of low concentrations of reactants (<1 M) gave a homogeneous reaction mixture. Therefore the most suitable procedure for bro- mination of the enamine I to the bromomethyl ketone $V$ was to use a type of "flow" reactor described below.

The yield of $\mathrm{V}$ determined by NMR analysis of the distilled crude product was $57 \%$ calculated from the amount of starting enamine. A disadvantage is that the yield of the enamine when prepared according to Ref. 13 is only $60-70 \%$ and use of more forced conditions in the synthesis of the enamine results in mixtures of tautomeric enamines. Thus the actual yield of the bromoketone calculated from the starting ketone is $30-40 \%$. A considerable advantage is that the product contains only trace amounts of the isomeric monobromo ketone IV. The desired bromomethyl ketone can easily be separated from unbrominated and dibrominated ketones by distillation.

\section{EXPERIMENTAL}

The enamines were prepared according to White and Weingarten. ${ }^{13}$ Separation of the isomers was conducted according to Pocar et al. ${ }^{10}$ NMR-spectra were recorded on a Varian A-60A or a JEOL C-60 HL spectrometer.

Procedure for the experiments in Table 1. 10 mmol of the enamine in $20 \mathrm{ml}$ of methylene chloride was added in one portion with vigorous stirring to a solution of the calculated amount of bromine in $20 \mathrm{ml}$ of methylene chloride. After one minute the reaction was quenched with 20 $\mathrm{ml}$ of water and the mixture subsequently stirred for $1 \mathrm{~h}$ to complete the hydrolysis. The organic layer was separated and washed with $10 \mathrm{ml}$ of $2 \% \mathrm{NaHSO}_{3}$-solution and $2 \times 10 \mathrm{ml}$ of water and then dried over anhydrous mag. nesium sulphate. The filtered solution was analyzed by GLC, using a PYE M 64 Gas Chromatograph with a FID and $270 \mathrm{~cm} \times 6 \mathrm{~mm}$, $12 \%$ QF-1 column at $120^{\circ} \mathrm{C}$. Chromatogrammes were recorded on a Philips recorder with a Disc integrator, and the relative amounts of the various ketones were determined from the integrals. Corrections were made for different FID responses of the various ketones. The peaks in the chromatogrammes were well resolved. The figures in Table 1 are the average values of three GLC determinations and the accuracy is within $\pm 1 \%$. The identities of the ketones were determined by means of GLC-MS, using a PYE M 84 Gas Chromatograph with a FID and an LKB 9000 Mass Spectrometer. Mass spectral analysis was aided by comparison with authentic sample spectra.

Procedure for the experiments in Table 2. 10 mmol of the enamine in $10 \mathrm{ml}$ of methylene chloride was added to a stirred solution of 10 
mmol of bromine in $90 \mathrm{ml}$ of methylene chloride. $5 \mathrm{ml}$ aliquots were hydrolyzed with $5 \mathrm{ml}$ of water and analyzed by GLC.

Preparation of bromomethyl isopropyl ketone. A typical procedure was: $39.0 \mathrm{~g}(0.454 \mathrm{~mol})$ of methyl isopropyl ketone and $120 \mathrm{ml}$ of morpholine were dissolved in $1000 \mathrm{ml}$ of isopentane. The mixture was cooled to $-5^{\circ} \mathrm{C}$ and a solution of $25 \mathrm{ml}(0.227 \mathrm{~mol})$ of titanium tetrachloride in $100 \mathrm{ml}$ of isopentane was added with stirring. The reaction mixture was stirred at $-5{ }^{\circ} \mathrm{C}$ overnight and filtered. Evaporation of the solvent at reduced pressure afforded the crude enamine $I$ almost free of the other isomer. The yield of the enamine was $44.9 \mathrm{~g}(64 \%)$ estimated from the NMR spectrum. The crude enamine was dissolved in $500 \mathrm{ml}$ of methylene chloride and transferred to a graduated dropping funnel. An equivalent amount of bromine was dissolved in $500 \mathrm{ml}$ of methylene chloride and subsequently transferred to a second graduated dropping funnel. The reagents were mixid in the following way. The two dropping funnels were mounted on a $250 \mathrm{ml}$ fournecked flask fitted with a mechanical stirrer, drying tube and an outlet stopcock from the bottom of the flask. The flask was cooled with dry ice. ethanol. (The cooling device was actually a plastic bag tied around the bottom outlet pipe with copper wire, the plastic bag being supported by a polystyrene foam beaker, cut into two halves and reassembled around the bag). The bromine solution was added as $25 \mathrm{ml}$ aliquots to the reaction flask with stirring and cooling. Each addition was followed by a rapid addition of a $25 \mathrm{ml}$ portion of enamine solution. The resulting mixture was stirred for an additional $30-40 \mathrm{~s}$. Thereafter it was tapped off via the bottom outlet pipe into a 21 threenecked flask, fitted with a stirrer, where it was quenched with a $25 \mathrm{ml}$ portion of water. A small amount of approximately $10 \% \mathrm{NaHSO}_{3}$-solution was added portionwise to the hydrolysate to destroy excess of bromine. After the reaction was complete, stirring with water was continued for another $40 \mathrm{~min}$. The organic layer was separated and the aqueous layer extracted with $100 \mathrm{ml}$ of methylene chloride. The combined organic layers were then washed three times with 200 $\mathrm{ml}$ of water and dried over anhydrous magnesium sulphate. The solvent was evaporated at reduced pressure, and the residue was distilled "bulb to bulb" at a reduced pressure. A final fractionation with a Vidmer column afforded $22.0 \mathrm{~g}$ of pure (>95\%) bromomethyl isopropyl ketone, $46 \%$ yield calculated on the starting enamine.

\section{REFERENCES}

1. Pedersen, R. L., Johnson, I. L., Holyz, R. P. and Ott, A. C. J. Amer. Chem. Soc. 79 (1957) 1115 .
2. Kuehne, M. E. J. Amer. Chem. Soc. 83 (1961) 1492.

3. Speziale, A. J. and Smith, L. R. J. Org. Chem. 28 (1963) 3492.

4. Paul, L., Schuster, E. and Hilgetag, G. Chem. Ber. 100 (1967) 1087.

5. Ahlbrecht, A. and Reiner, M. T. Tetrahedron Lett. (1971) 4901 .

6. Duhamel, P., Duhamel, L., Collet, C. and Haider, A. C. R. Acad. Sci. Ser. C 273 (1971) 1461.

7. Duhamel, L., Duhamel, P. and Poirier, J.-M. Tetrahedron Lett. (1973) 4237.

8. Keana, J. F. and Schumacher, R. P. Tetrahedron 26 (1970) 5191.

9. Coppens, W. and Schamp, N. Bull. Soc. Chim. Belg. 81 (1972) 643.

10. Pocar, D., Stradi, R. and Bianchetti, G. Gazz. Chim. Ital. 100 (1970) 1135.

11. Rappe, C. and Sachs, W. H. J. Org. Chem. 32 (1967) 3700.

12. Gaudry, M. and Marquet, A. Tetrahedron 26 (1970) 5611.

13. White, W. A. and Weingarten, H. J. Org. Chem. 32 (1967) 213.

14. Brändström, A. and Gustavii, K. Acta Chem. Scand. 23 (1969) 1215.

Received May 7, 1974. 